\title{
THE ROLE OF THE MODERN INFORMATICS SYSTEMS IN ASSISTED DECISIONS
}

\author{
Robert Gabriel DRAGOMIR \\ Spiru Haret University, \\ E-mail: robert73d@yahoo.com
}

\begin{abstract}
The present paper speaks about the complexity of the decisional phenomenon. First of all, we referred to the challenges of the informal society; strategic management is the one that occupies by them. Then we noticed that the most modern informational instruments are used by the strategic management in order to gain the best analysis and results. After that, we made references to the following solution of business intelligence: data warehouse, data cube OLAP and data mining. We presented their characteristics, advantages and disadvantages of usage one or another. In the end, we draw at the conclusions: the society has good premises of development by using such instruments.
\end{abstract}

Keywords: decisional phenomenon, management informatics system, data warehouse, OLAP cube

JEL Classification: C81

\section{Literature review}

The special literature treats the problem under discussion as being of a great importance within the scientific environment. This interests stands for the complexity of the present decision process. This implies the need to support the decision with the help of the performing informatics tools that are able to keep great volume of data based on accurate past experiments, inquire the great amount of information as the data type and offer scenarios for the future development of business.

Thus:

- The new vision of the intelligent business is (http://searchdatamanagement.techtarget.com): "Business intelligence (BI) is a technology-driven process for analysing data and presenting actionable information to help corporate executives, business managers and other end-users make more informed business decisions."

- The informatics solutions for assisted decisions speaks about (Maria Sueli Almeida, et al., 1999, p. 26): "Business intelligence tools can be broken down into three categories:

$\circ$ query and reporting;

○ online analytical processing (OLAP);

o information mining." 
- The main producers of platforms capable to offer intelligent assisted solutions are the following: Microsoft and Oracle.

- Microsoft offers support with SQL Server 2014 (http://www.microsoft.com) "SQL Server 2014 makes it easier and more cost effective to build high-performance, mission-critical applications, enterprise ready Big Data assets, and BI solutions that help employees make better decisions, faster. These solutions have the flexibility of being deployed on premises, in the cloud or in a hybrid environment, and can be managed through a common and familiar tool set."

○ The Oracle Solution is Oracle Database 12c (www.oracle.com) "Oracle Database 12c introduces a new multitenant architecture that makes it easy to consolidate many databases quickly and manage them as a cloud service. Oracle Database $12 \mathrm{c}$ also includes in-memory data processing capabilities delivering breakthrough analytical performance. Additional database innovations deliver new levels of efficiency, performance, security, and availability".

\section{Introduction}

The modern informatics systems assure the technological support for the present informational society. The complexity of the decisional phenomenon is due to the great data volume and the abstract and suggestive form of any report. So, the contribution of the informatics systems for the management is substantial. They can generate from simple routine abstracts, to results of special situations generated on years, terms, semesters, months, weeks, days. Different special type of information required for strategic business decisions can be obtained using marketing research techniques. The widespread use of information technology and modern telecommunications enable different organizations to implement new ways of marketing research and collection of data techniques using internet based platforms in order to gather the information needed. (Bondrea A., et al., 2014, p. 119)

The strategic management has to be sustained by the informatics systems at the level of planning, control and decision-making level. Their essential role is to filter the information in an intelligent manner and to present it in a concise form, which intuitively orient towards correct and efficient decisional solutions.

The management informatics system (Doina Fusaru, Zenovic Gherasim, 2008, p. 56) (MIS) monitors and recovers data within the socio and economic environment, picks data from the company transactions and operations, filters and selects data and presents them to the managers as pieces of information. The necessity for MIS consists in the fact that the managers need to be informed on different levels of the company.

The informatics systems for assisted decision (SIAD) answer directly to the investigations as concern the causes of some situations, indicating ways of action, means of resolving, methods of analysis, but unimposing decisions for the structured or unstructured decisional environment. Their capacity consists in receiving and processing external data. Unlike the management informatics systems, that operated initially with data and the relations between them, SIADs 
starts from managers to decision. The perspectives of the two types of informatics systems are different.

The solutions of business intelligence are to improve the processes of data analysis. The information is organized into structures that satisfy the needs of complete and complex analysis of data.

\section{Data Warehouse}

The concept of data warehouse (Nagabhushana S., 2006) was invented by Bill Inmon in 1990; he defined it as follows: a deposit is an oriented subject, integrated in time and of collecting the non-volatile data in assisting the decision of managing the process of decision assistance.

Data Warehouse is an assembly if data specially produced, in order to sustain the managerial decision. (Patil Preeti S., Rao Srikantha, Patil Suryakant B., 2011, pp. 33-37) Another definition of the data warehouse makes references on their provenience from different sources (distinct data bases with different structures). Data gathered from different sources, put in different places are usually stocked in one place, with a unified structure and typically resumed. This internal means of organisation allows an easier analysis of the data carried by extended companies. A data warehouse is considered a key factor of a decisional support system (DSS). The DSS have as an objective assisting the managerial process on different levels of decision, being usually the middle and strategic level, taking data from management information systems (MIS). (Fusaru D., 2004, p. 419)

Figure no. 1. Data Warehouse Architecture

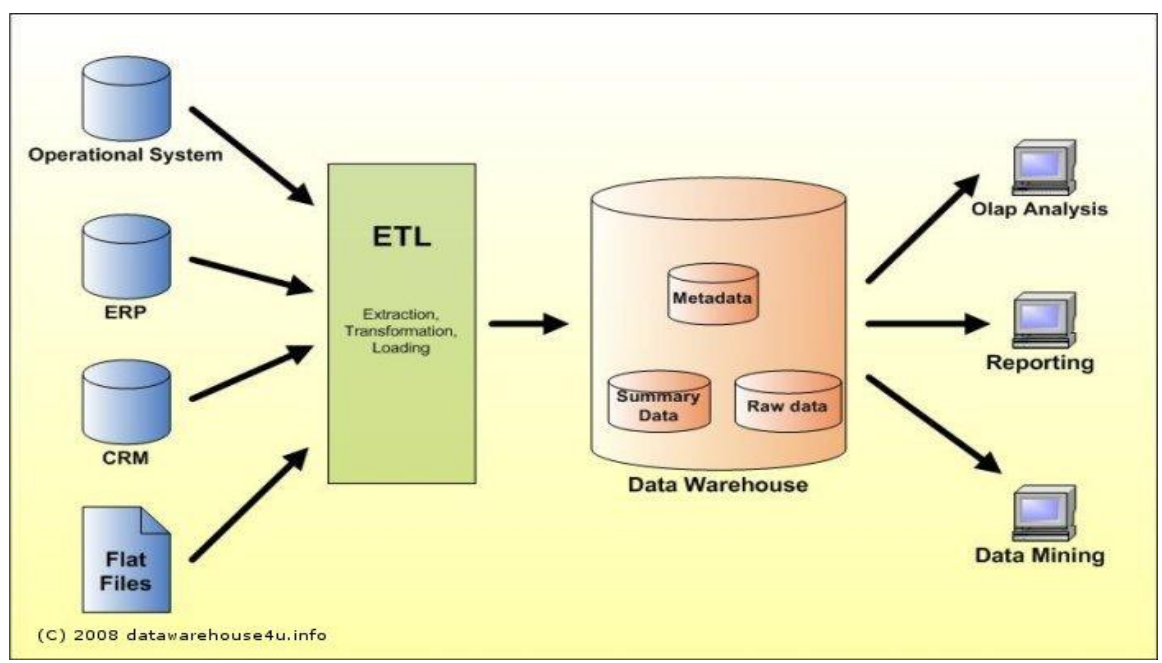

Source: http://datawarehouse4u.info/index_en.html

The data warehouses, the multidimensional organization or the data mining tools are but a few examples of technology capable to offer functions of detailed analysis. 
A data warehouse represents a database that is separately kept from the other operational databases of the company. Data in the source systems are extracted, cleaned, transformed and stocked in special warehouses in order to help the decisional process.

The data sources for the deposits are the following: current operational databases, old, archived databases and the external databases. To implement databases gives more strategic value to the companies. They also contribute to the costs reduction.

Dimensional modelling is a technique that allows conceptualisation and representation of the quantitative, measurable aspects of the activity, tight connected to its context. (Zaharie D., et al., 2001, p. 94)

Within the multidimensional model, one can meet several object types, having a great importance when analysing:

Dimensions - represent compound structures, attributes structured on different hierarchical levels. The OLAP council defines the concept of dimension as being a structured attribute of a cube, consisting in a list of members, seen by the users as being of the same type (as examples all months, trimesters, semesters, years represent the dimension of Time). (http://altaplana.com/olap/glossary.html) Dimension represents a very concise, intuitive manner of organizing and selection of data for exploring and analysis.

Facts tables are central tables. They contain attributes as of measure type and external keys towards tables' dimensions. The facts are usually numerical data that can be summed up and analysed at different levels.

Metadata represent the most important component of the data warehouse. In order to use the data warehouse, users have to know the type of data they can find in; metadata are not but data about data, data that describe the content of the warehouse.

The scheme of the data warehouse is a collection of objects, including tables, visions, indices and synonyms. There are several types of schemes used in their multidimensional modelling. The star scheme is the most frequent used model of organising the data warehouses.

The table containing facts stands for the central position and it is connected to the corresponding dimension tables.

A simple star scheme consists in one table facts and more tables of dimension. Here dimensions do not bare names; they contain redundant data that eliminate the necessity of the multiple connections between tables. There is only one connection in a star scheme, the one between the table facts and dimensions.

\section{Data cube OLAP}

Data warehousing and on-line analytical processing (OLAP) are essential elements of decision support, which has increasingly become a focus of the database industry. (Chaudhuri Surajit, Dayal Umeshwar, 1997, pp. 517-526)

Data warehousing technology is becoming essential for effective business intelligence, business strategy formulation and implementation in a globally 
competitive environment, wherein larger and larger amounts and date (doubling and 18 months) are required to be processed faster and faster (in a few seconds) for comprehension of its real mining and impact. (Prabhu C.S.R., 2008, p. 1)

OLAP (Online Analytical Processing) is the technology behind many Business Intelligence (BI) applications. OLAP is a powerful technology for data discovery, including capabilities for limitless report viewing, complex analytical calculations, and predictive "what if" scenario (budget, forecast) planning. (http://olap.com/olap-definition/)

OLAP functionality is characterized by dynamic multi-dimensional analysis of consolidated enterprise data supporting and user analytical and navigational activities including: (http://www.moulton.com/olap/olap.glossary.html)

- calculations and modelling applied across dimensions, through hierarchies and/or across members;

- trend analysis over sequential time periods;

- slicing subsets for on-screen viewing;

- drill-down to deeper levels of consolidation;

- reach-through to underlying detail data;

- rotation to new dimensional comparisons in the viewing area.

OLAP (On-line Analytical Processing) is characterized by relatively low volume of transactions. (http://datawarehouse4u.info/OLTP-vs-OLAP.html) Queries are often very complex and involve aggregations. For OLAP systems, a response time is an effectiveness measure. OLAP applications are widely used by Data Mining techniques. In OLAP database there is aggregated, historical data, stored in multidimensional schemas (usually star schema).

Within the multi-dimensional analysis, the cube with more than three dimensions is named n-dimensional cube or hypercube. The OLAP council defines it as being a group of data cells arranged after the data dimensions. A tridimensional matrix can be seen as a cube where every dimension forms a cube face. On-Line Analytical Processing is a type of technology used for organizing the great databases; it also offers decisional support for business. OLAP databases are split into several cubes, every cube is projected by a cube administrator, in order one can easy use the necessary PivotTable and PivotChart reports.

OLAP applications have to assure the users a multi-dimensional vision over data. The multi-dimensional operations implemented in the multi-dimensional model are:

- Drill Down and Roll Up represent operations of browsing within the hierarchies of the dimensions by aggregation at the superior levels or detailing at the inferior ones.

- Rotations represent the most used operations for the multi-dimensional data structures and offer the user the chance of choosing the perspective on data.

- Sections represent visions or images (views), specific to different categories of users, by operations of sectioning, thus obtaining bi-dimensional slices.

The multi-dimensional modelling bases on a peculiar model, an own vision as concern requires of the multi-dimensional analysis, scientific language and formalism. 
There is not up to present any multi-dimensional data model unanimously accepted. We consider it necessary for serving as standardization and future research.

The conceptual modelling of the data warehouses starts from dimensional models that group data in the relational tables in star or snowflake schemes, where we can find the quantitative data in tables of transactions aggregated especially on time unity (day) and on other criteria (client, service, product etc). (http://datawarehouse4u.info/OLTP-vs-OLAP.html)

Creation of an OLAP cube bases the multi-dimensional data structure. These data are stocked in a multi-dimensional data structure. Here we define the data source of the table facts and dimensions. The measure is the result of some combinations between many columns, as expressions. The options for memorisation are:

- ROLAP data are still stored into the relational database, together with aggregations;

- MOLAP memorized both data and aggregations in multi-dimensional structures;

- HOLAP data are stored in the relational database, but aggregations are stored in multi-dimensional structures.

The implementations of the data servers for OLAP processing include the following types of servers:

$\checkmark$ ROLAP are intermediate servers that stand between the relational back-end servers and front-end client instruments. They use systems of relational or relational extended databases, including optimizations for every system of the type DBMS. As opposed to MOLAP, ROLAP technology tends towards a greater scalability.

$\checkmark$ MOLAP represents servers based on multi-dimensional visualisations of data, through stocking motors of the multi-dimensional tables. Data are seen as structures of table data, as data cubes. The advantage of using a data cube is that it allows a rapid indexation of centralized and pre-calculated data. Many servers of MOLAP type stock data by using two levels: sets of organized data and sets of dispersed data. The more dense sub-cubes are identified and stocked as table structures, while the dispersed sub-cubes imply compressing technologies for an efficient way of stocking.

$\checkmark$ HOLAP integrates the advantages of the two above-mentioned technologies: scalability and rapid indexation. This type of server allows the usage of great bulk of detailed data.

When speaking about the data warehouses, the more they are used, the more they evolve. This evolution takes place under stages. Initially, the data warehouses were used to generate reports and for answers to pre-definite interrogations. Progressively, they were used to analyse centralized and detailed data. Later on, they were improved with strategic and decisional competences, performing multidimensional analysis and complex operations as slice or dice. Nowadays, the data warehouses are implied in knowledge and strategic decision, using tools of data mining. Thus, the data mining tools can be grouped into access and regaining tools, tools for databases reporting, tools for data analysing and mining tools. 
We can distinguish three means of applications within the data warehouses:

$\checkmark$ Information processing (offers interrogations, basic statistical analyses, graphic reports, diagrams, tables; the present trend is represented by low price access instruments based on web technology, later on integrated into web browsers);

$\checkmark$ analytical processing (allows OLAP basic operations, including operations such as slice and dice, drill-down, roll-up, slewing; generally it operates with centralized historic or detailed data; the force of this on-line analytical processing consists in multi-dimensional analysis of data in the data warehouses;

$\checkmark$ data mining (discovers hidden model and associations, analytical models of building, classifications and performing forecasts and presents the results by using instruments of visualisation).

As concern data mining, consistent researches have been made on different platforms of data organisation: transactional databases, relational databases, spatial databases, base of text type etc.

The OLAM - On-Line Analytical Mining - named also OLAP mining integrates the analytical processing with data mining in multi-dimensional databases. Among many and different paradigms and architectures of the data mining systems, OLAM is extremely important, because it offers:

$\checkmark$ high quality of data in data warehouses. The most instruments operate with integrated, relevant and cleaned data that need stages for pre-processing for cleaning, integration and transformation. Such a data warehouse becomes a valuable data source for OLAP, but also for data mining process. Data mining can be used for data cleaning and integration.

$\checkmark$ data infrastructure processing. Information processing and data infrastructure analysis are built around data warehouses. They comprise accessing, integration, consolidation, transformation of the multiple heterogeneous data bases, facilitation when accessing and web services, reporting and OLAP analysis instruments.

$\checkmark$ analysis of data mining based on OLAP. Data processing requires a preliminary stage of data mining. Users usually want to select relevant recordings, analyse them on different levels of detailing or to present the final results under different forms. The on-line analytical processing offers facilitations for mining on different data sub-sets and on different levels of abstraction, using specific processes: drilling, filtering and slicing of a cube of data and of some intermediate mining results.

$\checkmark$ functions of mining the on-line selected data. There are many situations when the user does not know what kind of data he is to mine. By using the OLAP interrogation with multiple functions of data mining, the on-line analytical mining offers flexibility in order to select the desired mining functions for fulfilling the proposed objectives. 
Figure no. 2. Data Warehousing (OLAP) to Data Mining (OLAM)

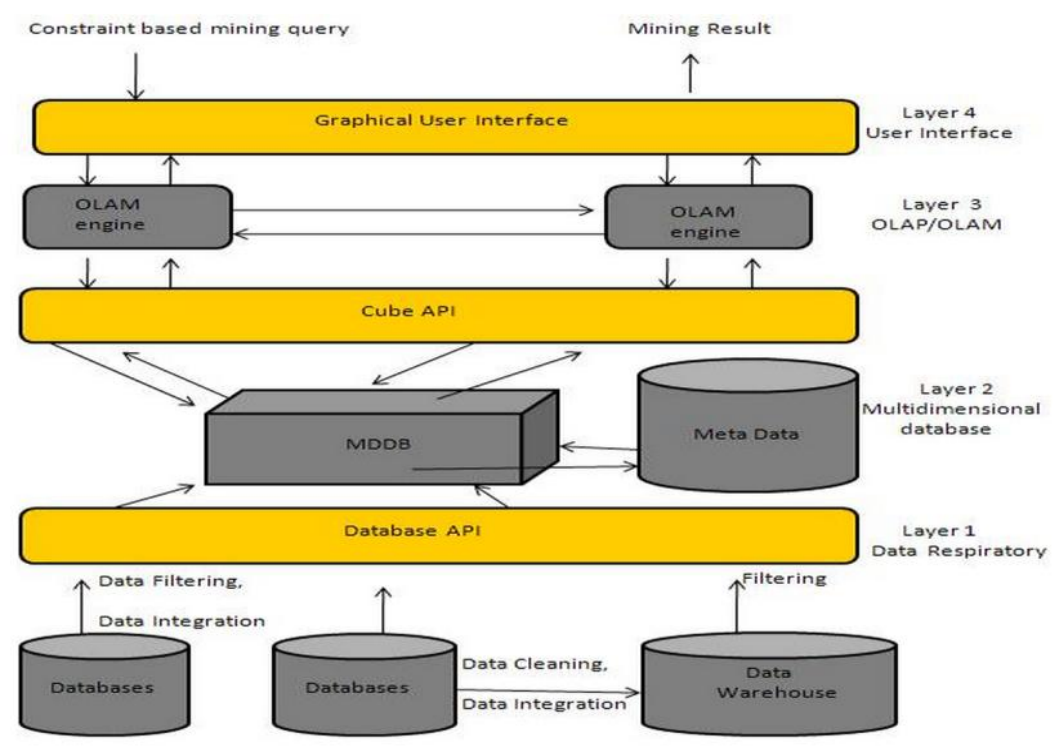

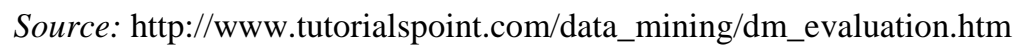

Online Analytical Mining integrates with Online Analytical Processing with data mining and mining knowledge in multidimensional databases. Here is the diagram that shows integration of both OLAP and OLAM, as can be seen in the figure above.

\section{Conclusions}

Each of the software solutions presented above is able to valorise information. The strategic decision bases on two main components: the former of them is the past experience that is valorised in a proper way by the data warehouse; the later occupies with the possibility to create scenarios, using the data. Scenarios lead to simulations of the intelligent business. These stand on the force of the OLAP instrument and also the capacity of data mining of creating many new scenarios.

Although the manager is the one who can decide, it is important for him to be sustained during this process by these performing tools.

This work was supported by the project "Excellence academic routes in doctoral and postdoctoral research - READ" co-funded from the European Social Fund through the Development of Human Resources Operational Programme 2007-2013, contract no. POSDRU/159/1.5/S/137926. 


\section{References}

1. Almeida Maria Sueli, Ishikawa Missao, Reinschmidt Joerg, \& Torsten Roeber (1999), Getting Started with Data Warehouse and Business Intelligence, IBM Corporation, International Technical Support Organization.

2. Bondrea A. Aurelian, Geangu Iuliana Petronela, Gârdan Daniel Adrian, \& Crețoiu Raluca (2014), 'Consumer rights protection in the context of online marketing research', Proceedings of the 4th International Conference on Law and Social Order, Constanta, 25 aprilie, vol. 2, New York: Addleton Academic Publishers, pp. 118-128

3. Chaudhuri Surajit, \& Dayal Umeshwar (1997), An Overview of Data Warehousing and OLAP Technology, Appears in ACM Sigmod Record, 26(1), pp. 517-526, http://research.microsoft.com/pubs/76058/sigrecord.pdf, accessed on 10 November, 2014.

4. Fusaru Doina, \& Gherasim Zenovic (2008), Informatică managerială, Bucureşti: Editura Fundaţiei România de Mâine.

5. Fusaru Doina, Gherasim Zenovic, Lungu Ion, \& Bâra Adela (2004), Tehnici şi arhitecturi pentru micşorarea timpului de răspuns în sistemele cu depozite de date (data warehouse) (Techniques and architectures for decreasing the response time in the date warehouse systems), Annals of Spiru Haret University, Economic Series, 4(4), pp. 419-426.

6. Nagabhushana S. (2006), Data Warehousing: OLAP and Data Mining, New Delhi: New Age International.

7. Patil Preeti S., Rao Srikantha, Patil Suryakant B. (2011), "Optimization of data warehousing system: Simplification in reporting and analysis." IJCA Proceedings on International Conference and workshop on Emerging Trends in Technology (ICWET), 9(6), pp. 33-37.

8. Prabhu C.S.R. (2008), Data warehousing: concept, techniques, products and applications, $3^{\text {rd }}$ edition, PHI Learning Pvt. Ltd.

9. Zaharie D., Albescu F., Bojan I., Ivancenco V., Vasilescu C. (2001), Sisteme informatice de asistarea deciziei, Bucureşti: Editura Dual Tech.

10. http://altaplana.com/olap/glossary.html, accessed on 10 November 2014.

11. http://datawarehouse4u.info/index_en.html, accessed on 25 October 2014.

12. http://datawarehouse4u.info/OLTP-vs-OLAP.html, accessed on 25 October 2014.

13. http://www.microsoft.com/en-us/server-cloud/products/sql-server/, accessed on 13 November 2014.

14. http://www.moulton.com/olap/olap.glossary.html, accessed on 25 October 2014.

15. http://olap.com/olap-definition/, accessed on 25 October 2014.

16. https://www.oracle.com/database/index.html, accessed on 15 November 2014.

17. http://searchdatamanagement.techtarget.com/definition/business-intelligence, accessed on 15 November 2014.

18. http://www.tutorialspoint.com/data_mining/dm_evaluation.htm 
